\title{
Capacidade Soma para Canais de Acesso Múltiplo com T Usuários e N Freqüências.
}

\author{
Manish Sharma e Jaime Portugheis
}

\begin{abstract}
Resumo-Consideramos neste artigo o canal de acesso múltiplo MAC (do inglês Multiple Access Channel) ruidoso: um MAC não ruidoso com $T$ usuários e $N$ freqüências com conhecimento de intensidade concatenado com um canal ruidoso. Dada uma condição nas densidades de probabilidade de transição do canal ruidoso, a capacidade soma é atingida através de um produto de distribuições uniformes no alfabeto de entrada. Obtivemos resultados numéricos para o caso onde o canal ruidoso inclui desvanecimento Rayleigh e ruído aditivo Gaussiano branco (AWGN). Devido às dimensões das integrais envolvidas, um limitante superior foi considerado, sendo o seu valor muito próximo dos valores obtidos através de integração por Monte Carlo. Foi observado também que o MAC ruidoso se comporta de modo análogo a um canal MAC não ruidoso sem conhecimento de intensidade: há um valor de $T$ que maximiza a capacidade soma.
\end{abstract}

Palavras-Chave-Capacidade de Canal, Canais de Acesso Múltiplo.

Abstract-In this paper, we consider a noisy multiple access channel (MAC): the $T$-user $N$-frequency noiseless MAC with intensity information in cascade with a noisy channel. Given a condition on the transition probabilities of the noisy channel, the sum capacity of the noisy MAC is achieved by a product of identical uniform input distributions. Numerical results for the sum capacity are obtained for a channel that includes Rayleigh fading and Additive White Gaussian Noise (AWGN). Due to the dimension of the integrals involved, an upper bound was considered and found to be very close to the values obtained through Monte Carlo integration. Moreover, it was observed that the sum capacity of this noisy MAC behaves similarly to a noiseless MAC without intensity information: there is a value of $T$ that maximizes sum capacity.

Keywords - Channel Capacity, Multiple Access Channel.

\section{INTRODUÇÃO}

Uma classe de MAC com $T$ usuários e $N$ freqüências foi investigada em [1]. Dois canais específicos foram estudados. Em ambos os modelos, os $T$ usuários tinham um alfabeto de entrada em comum com $N$ freqüências. Entretanto, os alfabetos de saída eram diferentes. Para o canal sem conhecimento de intensidade, o símbolo de saída era binário e indicava somente se uma freqüência de entrada estava sendo utilizada ou não. Este canal é referido como canal A. Para o canal com conhecimento de intensidade, o símbolo de saída era um número inteiro entre 0 e $T$ e indicava quantos usuários estavam utilizando uma certa freqüência de entrada. Este canal

\footnotetext{
Departamento de Comunicações, Faculdade de Engenharia Elétrica, Universidade Estadual de Campinas, E-mails: [sharma][jaime]@decom.fee.unicamp.br . Este trabalho foi parcialmente financiado pela FAPESP (06/00330-7).
}

é referido como canal B. Em ambos os modelos assumiuse que não ocorriam erros devido a ruído ou outras fontes externas.

Neste artigo, consideramos a presença de erros, modelando o canal MAC ruidoso como a concatenação de um canal B com um canal ruidoso. Para avaliar o desempenho deste MAC ruidoso, consideramos a capacidade soma, um limitante superior para a soma das taxas de todos os usuários. Mostramos que, para qualquer MAC com $N$ freqüências onde uma permutação de índices não altera as estatísticas do canal, um produto de distribuições uniformes de entrada atinge a capacidade soma.

Após, consideramos a capacidade soma para um MAC ruidoso que inclui desvanecimento Rayleigh e AWGN. Devido às dimensões das integrais envolvidas, é computacionalmente complicado avaliar numericamente as integrais envolvidas. É necessário utilizar integração por Monte Carlo. Entretanto, os valores de um limitante superior simples são bem próximos dos valores encontrados através das integrais por Monte Carlo.

Em alguns sistemas de acesso múltiplo, em vez de realizar a detecção conjunta das mensagens transmitidas por todos os usuários, o receptor pode detectar somente a mensagem de um único usuário. Isto é normalmente denominado deteç̧ão de um único usuário SUD (do inglês Single User Detection). Para este receptor mais simples, nós definimos a capacidade soma e a comparamos com os valores obtidos para o MAC ruidoso.

Este artigo está organizado da seguinte forma: na Seção II apresentamos um modelo para o canal ruidoso que inclui desvanecimento Rayleigh e AWGN. A capacidade soma para o MAC é estudada na Seção III. Na Seção IV consideramos o receptor SUD. Na Seção V analisamos alguns resultados numéricos, e na Seção VI apresentamos as nossas considerações finais.

\section{CAnal de Acesso Múltiplo Ruidoso}

O modelo para o canal MAC considerado é baseado no diagrama da figura 1. O canal ruidoso, $p(\mathbf{R} / \mathbf{c})$, é derivado do modelo descrito em [2].

Há $T$ usuários. Num dado instante, cada um deles independentemente escolhe uma mensagem dentre um alfabeto com $N$ símbolos. O símbolo escolhido pelo $j$-ésimo usuário é $m^{j}$, um inteiro entre 1 e $N$. As mensagens escolhidas por todos os usuários podem ser representadas por um vetor $\mathbf{M}=\left\{m^{1}, m^{2}, \ldots, m^{T}\right\}$. Cada símbolo está associado a um chip, uma banda no espectro de frequiências. Cada mensagem $m^{j}$ é convertida num vetor $\mathbf{c}^{j}$ com dimensão $N$, dado por: 


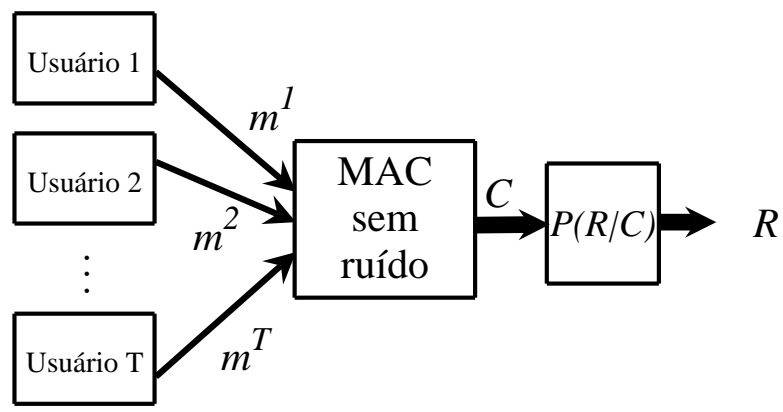

Fig. 1. Canal de Acesso Múltiplo Ruidoso

$$
c_{n}^{j}= \begin{cases}1, & \text { se } m^{j}=n \\ 0, & \text { caso contrário }\end{cases}
$$

O elemento $c_{n}^{j}$ (um chip) está associado a sinais com duração $\tau$, transmitidos com energia $E_{c}=S \tau$. O canal inteiro tem banda $1 / \tau$, dividido em $N$ sub-bandas (chips) com largura $1 / N \tau$. A energia por chip está associada com a energia por bit através da igualdade $E_{c}=E_{b} \log _{2} N$.

Definimos as seguintes formas ortogonais básicas, com duração $\tau$ :

$$
\begin{aligned}
& x_{n}(t)=\sqrt{2 S} \cos 2 \pi\left[f_{0}+(n-1) / \tau\right] t, \\
& y_{n}(t)=\sqrt{2 S} \sin 2 \pi\left[f_{0}+(n-1) / \tau\right] t,
\end{aligned}
$$

onde $f_{0}$ é uma freqüência base e $n=1,2, \ldots, N$.

$\mathrm{O}$ sinal que o $j$-ésimo usuário transmite no $n$-ésimo chip é então dado por:

$$
s_{n}^{j}(t)=c_{n}^{j} x_{n}(t)
$$

Inicialmente, podemos considerar que todos os usuários transmitem com a mesma fase. Na recepção, cada usuário sofre um desvanecimento Rayleigh $\alpha_{n}^{j}$ e um atraso de fase aleatório $\phi_{n}^{j}$ (uniformemente distribuído), todos estatisticamente independentes entre si. Há também uma única componente de ruído branco Gaussiano aditivo $n(t)$ com densidade bilateral $N_{0} / 2$, de modo que o sinal recebido resultante é:

$$
r_{n}(t)=\sum_{j=1}^{T}\left\{c_{n}^{j} \alpha_{n}^{j}\left[\cos \phi_{n}^{j} x_{n}(t)+\sin \phi_{n}^{j} y_{n}(t)\right]\right\}+n(t)
$$

A energia de cada chip é detectada através de filtros casados:

$$
\begin{aligned}
& X_{n}=\frac{1}{E_{c}} \int_{0}^{\tau} r_{n}(t) x_{n}(t) d t=\kappa_{n}+\sum_{j=1}^{T} c_{n}^{j} \alpha_{n}^{j} \cos \phi_{n}^{j} \\
& Y_{n}=\frac{1}{E_{c}} \int_{0}^{\tau} r_{n}(t) y_{n}(t) d t=\lambda_{n}+\sum_{j=1}^{T} c_{n}^{j} \alpha_{n}^{j} \sin \phi_{n}^{j}
\end{aligned}
$$

onde $\kappa_{n}$ e $\lambda_{n}$ são variáveis aleatórias Gaussianas correspondentes ao ruído, com média zero e variância igual a $d / 2$, $d=N_{0} / E_{c}$. Podemos mostrar que as saídas dos filtros $X_{n}$ e $Y_{n}$ são descorrelacionadas.

Seja $c_{n}=\sum_{j=1}^{T} c_{n}^{j}$ o número de usuários que estão utilizando o $n$-ésimo chip. Cada um dos $\alpha_{n}^{j} \cos \phi_{n}^{j}$ ou $\alpha_{n}^{j} \sin \phi_{n}^{j}$ é uma variável Gaussiana com média zero e variância igual a $1 / 2$. Logo, $X_{n}$ e $Y_{n}$ são variáveis Gaussianas independentes com média zero e variância igual a $\left(c_{n}+d\right) / 2$.

A saída para o $n$-ésimo chip é obtida somando-se o quadrado de $X_{n}$ e $Y_{n}$ :

$$
R_{n}=X_{n}^{2}+Y_{n}^{2}
$$

A distribuição de $R_{n}$, condicionada ao número de usuários ativos no $n$-ésimo chip, é exponencial com parâmetro $c_{n}+d$ :

$$
p\left(R_{n} \mid c_{n}\right)=\frac{\exp -\frac{R_{n}}{c_{n}+d}}{c_{n}+d}
$$

Assumindo que todos os usuários utilizam a mesma distribuição de entrada, o número de usuários ativos por chip tem uma distribuição binomial, dada por:

$$
P\left(c_{n}\right)=B\left(c_{n}, T, \mu_{n}\right)=\left(\begin{array}{c}
T \\
c_{n}
\end{array}\right) \mu_{n}^{c_{n}}\left(1-\mu_{n}\right)^{T-c_{n}}
$$

onde $\mu_{n}$ é a probabilidade de um usuário utilizar a $n$-ésima frequiência.

É possível agora escrever uma equação que descreve a densidade de probabilidade de $R_{n}$, incondicionado à $c_{n}$ :

$$
P\left(R_{n}\right)=\sum_{c_{n}=0}^{T} P\left(c_{n}\right) p\left(R_{n} \mid c_{n}\right)
$$

Dadas as mensagem de todos os usuários, os valores de $R_{n}$ são estatisticamente independentes, e podemos escrever a seguinte equação para a densidade de probabilidade de $\mathbf{R}=$ $\left\{R_{1}, R_{2}, \ldots, R_{N}\right\}$ :

$$
p(\mathbf{R} \mid \mathbf{M})=p(\mathbf{R} \mid \mathbf{c})=\prod_{n=1}^{N} p\left(R_{n} \mid c_{n}\right)
$$

onde $\mathbf{c}=\left\{c_{1}, c_{2}, \ldots, c_{N}\right\}$ é uma função determinística de $\mathbf{M}$.

Entretanto, incondicionado aos valores das mensagens, os valores de $R_{n}$ não são estatisticamente independentes. Podemos escrever a seguinte equação para descrever a densidade de probabilidade do vetor $\mathbf{R}$, com dimensão $N$ :

$$
P(\mathbf{R})=\sum_{\mathbf{c} \in \Gamma(\mathbf{c})} P(\mathbf{c}) \prod_{n=1}^{N} p\left(R_{n} \mid c_{n}\right)
$$

onde $\Gamma(\mathbf{c})$ é o conjunto de todos os possíveis valores para $\mathbf{c}$ tais que $\sum_{i=1}^{N} c_{n}=T$. 


\section{CAPACIDADE SOMA}

A capacidade soma, $C_{S}$, é dada por:

$$
C_{S}=\max \mathcal{I}(\mathbf{M}, \mathbf{R})=\max \{\mathcal{H}(\mathbf{R})-\mathcal{H}(\mathbf{R} \mid \mathbf{M})\}
$$

onde a maximização é feita sobre as distribuições de entrada dos usuários. Em [1], foi mostrado que a distribuição ótima para o MAC não ruidoso com conhecimento de intensidade ocorre quando todos os usuários utilizam a distribuição uniforme sobre as $N$ freqüências. No Apêndice I, mostramos que o mesmo produto de distribuições uniformes atinge a capacidade soma para canais MAC ruidosos com densidades de probabilidade de transição dadas pela equação (7). Além disso, mostramos que este resultado pode ser estendido para qualquer MAC com $N$ sinais ortogonais onde uma permutação de índices não altera as densidades $\left\{p\left(R_{n} \mid c_{n}\right)\right\}$.

Podemos escrever $\mathcal{H}(\mathbf{R} \mid \mathbf{M})$ de uma forma fechada, utilizando:

$$
\mathcal{H}(\mathbf{R} \mid \mathbf{M})=\mathcal{H}(\mathbf{R} \mid \mathbf{c})=\sum_{n=1}^{N} \mathcal{H}\left(R_{n} \mid c_{n}\right)
$$

Como todos os chips tem a mesma distribuição de entrada e $P\left(c_{n}\right)$ é dada pela equação (8), temos:

$$
\mathcal{H}(\mathbf{R} \mid \mathbf{M})=\frac{N}{\log 2} \sum_{c=0}^{T} B(c ; T ; \mu)[1-\log (c+d)]
$$

onde $\mu=1 / N$.

Entretanto, não parece ser possível obter uma forma fechada para $\mathcal{H}(\mathbf{R})$, devido à complexidade de $p(\mathbf{R})$. Para contornar este problema, utilizamos o método de integração por Monte Carlo.

\section{A. Integração pelo método Monte Carlo}

Para realizar a integração por Monte Carlo, é necessário gerar vários valores aleatórios para $\mathbf{R}$ (de acordo com a sua distribuição estatística) e avaliar $p(\mathbf{R})$ para cada valor gerado. A forma como $p(\mathbf{R})$ foi escrita na equação (11) não é apropriada para computação pois o número de termos do somatório é próximo a $N^{T}$. Reescrevendo (11), obtemos:

$$
\begin{aligned}
p(\mathbf{R})= & \sum_{\mathbf{c} \in \Gamma(\mathbf{c})} P(\mathbf{c}) \prod_{n=1}^{N} p\left(R_{n} \mid c_{n}\right) \\
= & \sum_{c_{1}=0}^{T}\left\{P ( c _ { 1 } ) p ( R _ { 1 } | c _ { 1 } ) \sum _ { c _ { 2 } = 0 } ^ { T } \left\{P_{2}\left(c_{2} \mid c_{1}\right) p\left(R_{2} \mid c_{2}\right)\right.\right. \\
& \left.\times\left\{\cdots \sum_{c_{N}=0}^{T}\left\{P_{N}\left(c_{N} \mid \sum_{i=1}^{N-1} c_{i}\right) p\left(R_{N} \mid c_{N}\right)\right\} \cdots\right\}\right\}
\end{aligned}
$$

Esta equação pode ser calculada em $N$ passos, de acordo com as seguintes equações:

$$
\begin{gathered}
c_{i}^{\prime}=\sum_{i^{\prime}=1}^{i-1} c_{i^{\prime}} \\
P_{i}\left(c_{i} \mid c_{i}^{\prime}\right)=\left(\begin{array}{c}
T-c_{i}^{\prime} \\
c_{i} \\
c_{i}
\end{array}\right) \frac{1}{N-i+1}\left(1-\frac{1}{N-i+1}\right)^{T-c_{i}^{\prime}-c_{i}} \\
f_{i}\left(c_{i}^{\prime}, \mathbf{R}\right)=\sum_{c_{i}=0}^{T-c_{i}^{\prime}} P\left(c_{i} \mid c_{i}^{\prime}\right) p\left(R_{i} \mid c_{i}\right) f_{i+1}\left(c_{i}+c_{i}^{\prime}, \mathbf{R}\right) \\
f_{N}\left(c_{i}^{\prime}, \mathbf{R}\right)=\sum_{c_{i}=0}^{T-c_{N}^{\prime}} P\left(c_{i} \mid c_{N}^{\prime}\right) p\left(R_{N} \mid c_{N}\right) \\
P(\mathbf{R})=f_{1}(0, \mathbf{R})
\end{gathered}
$$

A função $f_{i}(\cdot)$ é definida para $i=1, \ldots, N$. Ela toma como argumento os valores de $R_{i}, \ldots, R_{N}$, e somente $R_{i}$ diretamente. Os valores de $P_{i}$ precisam ser calculados somente uma vez. Os cálculos começam com $i=N$. Há $N$ somatórios com no máximo $T$ termos cada.

\section{B. Limitante Superior para a Capacidade Soma}

Um limitante superior simples para $\mathcal{H}(\mathbf{R})$ pode ser obtido considerando que os valores de $R_{i}$ são estatisticamente independentes. Neste caso:

$$
\mathcal{H}(\mathbf{R}) \leq-N \int_{0}^{\infty} P\left(R_{n}\right) \log _{2} P\left(R_{n}\right) d R_{n}
$$

já que todos os $R_{i}$ tem a mesma distribuição.

\section{Caso não Ruidoso}

Para o canal $\mathrm{B}, \mathbf{R}=\mathbf{c}$, i.e., $\mathcal{H}(\mathbf{R} \mid \mathbf{M})=0$. A capacidade soma é reduzida a $\mathcal{H}(\mathbf{R})$, com expressão analítica:

$$
C=\sum_{\mathbf{c} \in \Gamma(\mathbf{c})} \frac{T !}{c_{1} ! c_{2} ! \ldots c_{N} !} \frac{1}{N^{T}} \log _{2}\left[\frac{N^{T}}{T ! / c_{1} ! c_{2} ! \ldots c_{N} !}\right]
$$

\section{DetecÇÃo de um Único Usuário}

Em [2], detecção por máxima verossimilhança foi feita para cada mensagem $m^{j}$. Isto é, o receptor detecta um único usuário (SUD) em vez de realizar a deteç̧ão conjunta de todas as mensagens $\left\{m^{1}, m^{2}, \ldots, m^{T}\right\}$. A taxa máxima na qual qualquer usuário pode transmitir informações corretamente é $\mathcal{I}\left(m^{j}, \mathbf{R}\right)$, que é a mesma para todos os usuários. Esta taxa é dada por:

$$
C_{S U D}=\max I\left(\mathbf{R}, m^{j}\right)=\max \left[\mathcal{H}(\mathbf{R})-\mathcal{H}\left(\mathbf{R} \mid m^{j}\right)\right]
$$

onde a maximização é feita sobre a distribuição de probabilidade de $m^{j}$. No Apêndice II mostramos que a distribuição uniforme atinge $C_{S U D}$.

A capacidade soma é então dada por $T \cdot C_{S U D}$. Este valor pode ser comparado com o valor $C_{S}$ obtido para o caso MAC.

O cálculo de $\mathcal{H}(\mathbf{R})$ já foi discutido. Não há um forma fechada para $\mathcal{H}\left(\mathbf{R} \mid m^{j}\right)$. Não podemos considerar que, dado $m^{j}$ (ao contrário de $\mathbf{M}$ ), os valores de $R_{n}$ são estatisticamente 
independentes. Entretanto, assim como para $\mathcal{H}(\mathbf{R})$, podemos realizar integração por Monte Carlo. Podemos avaliar $p\left(\mathbf{R} \mid m^{j}\right)$ usando a equação (15) e um subconjunto de $\Gamma(\mathbf{c})$ que contém todos os possíveis valores de $\mathbf{c}$ tais que a mensagem do $j$ ésimo usuário é $m^{j}$.

Em [3], os valores de $R_{n}$, dado $m^{j}$, foram considerados estatisticamente independentes. Esta suposição simplificou o cálculo das probabilidades de erro ao mesmo tempo em que forneceu uma boa aproximação para estes valores. Sob esta suposição, podemos obter um limitante superior para $\mathcal{H}\left(\mathbf{R} \mid m^{j}\right)$ (um limitante inferior para $C_{S U D}$ ) avaliando numericamente as seguintes integrais:

$$
\begin{gathered}
\mathcal{H}\left(\mathbf{R} \mid m^{j}\right) \leq \int_{0}^{\infty} p\left(R_{n} \mid m^{j}=n\right) \log _{2}\left[p\left(R_{n} \mid m^{j}=n\right)\right] d R_{n} \\
\quad+(N-1) \cdot \int_{0}^{\infty} p\left(R_{n} \mid m^{j} \neq n\right) \log _{2}\left[p\left(R_{n} \mid m^{j} \neq n\right)\right] d R_{n}
\end{gathered}
$$

As densidades de probabilidade $p\left(R_{n} \mid m^{j}\right)$ são descritas por:

$$
p\left(R_{n} \mid m^{j}\right)=\sum_{j=0}^{T-1} B\left(j, T-1, \mu_{n}\right) \frac{\exp \left(-\frac{R_{n}}{j+d+\delta_{n m^{j}}}\right)}{j+d+\delta_{n m^{j}}}
$$

onde $\delta_{n m^{j}}$ é o delta de Kronecker, valendo 1 se $n=m^{j}$ ou 0 caso contrário. Esta equação foi obtida em [2] considerando a transformada inversa de Fourier da função característica de $R_{n}$. Alternativamente, ela pode ser obtida usando equações 7 e 8 , considerando que o número de usuários transmitindo na $n$-ésima freqüência varia binomialmente entre $\delta_{n m^{j}}$ (se não há interferentes) e $T-1+\delta_{n m^{j}}$ (se todos os interferentes estão presentes).

\section{Resultados}

Resultados para a capacidade soma estão presentes nas figuras 2 a 4 para $N=8,16$ e 32 freqüências. Para obter linhas suaves, em torno de $10^{6}$ vetores aleatórios foram utilizados por ponto nas integrais por Monte Carlo. Também apresentamos, como parâmetro de comparação, a capacidade soma para o caso não ruidoso.

Podemos observar que o limitante superior para a capacidade soma está muito próximo aos resultados encontrados através de integrais de Monte Carlo. O limitante fica mais próximo com um aumento em $N, T$ ou $d$. Isto parece natural já que, com um aumento em $d$ (diminuição em $E_{b} / N_{0}$ ), os valores recebidos estão mais relacionados ao ruído do que aos sinais transmitidos pelos usuários. Para baixos valores de $T$ e $d$, a capacidade é próxima ao caso não ruidoso.

Também podemos observar que há um número ótimo de usuários que maximiza a capacidade, um resultado semelhante ao canal A em [1]. O valor máximo ocorre próximo ao máximo do limitante superior, servindo este de referência.

O limitante inferior para a capacidade soma $T \cdot C_{S U D}$ está próximo aos valores encontrados através das integrais por Monte Carlo. Assim para o caso MAC, o limitante fica melhor com um aumento em $T$.

Vale a pena ressaltar que alguns valores obtidos através das integrais por Monte Carlo podem estar abaixo dos valores

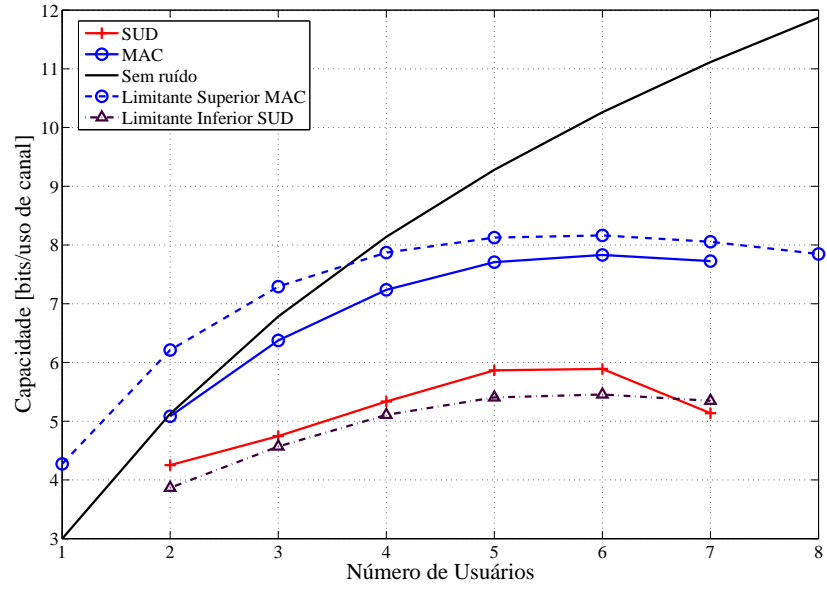

Fig. 2. Capacidade soma para $\mathrm{N}=8, E_{b} / N_{0}=25 d B$

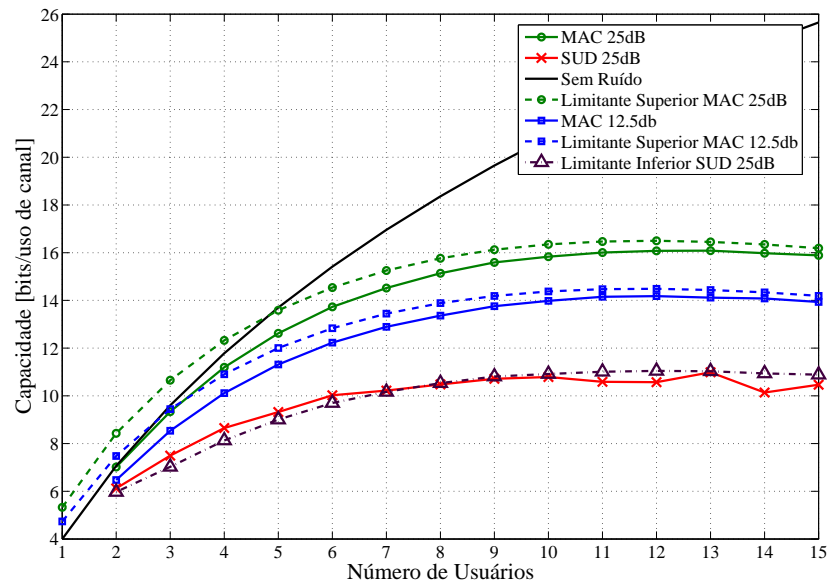

Fig. 3. Capacidade soma para $\mathrm{N}=16, E_{b} / N_{0}=12.5 d B$ and $25 d B$

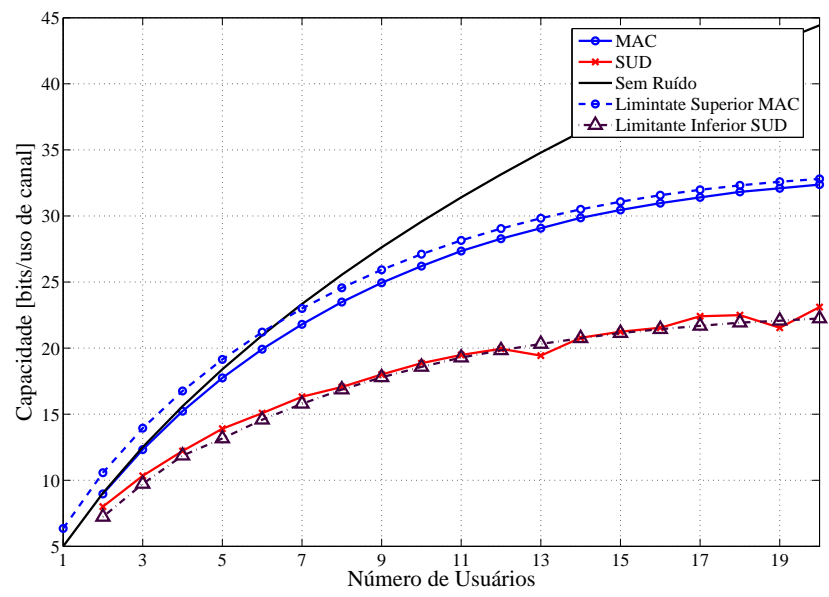

Fig. 4. Capacidade soma para $\mathrm{N}=32, E_{b} / N_{0}=25 d B$ 
obtidos para o limitante inferior. Esta discrepância é creditada ao número de amostras utilizadas para gerar cada ponto.

Como esperado, há um ganho significativo na capacidade soma ao se realizar a detecção conjunta das mensagens.

\section{CONCLUSÃO}

Neste artigo apresentamos alguns resultados para canais de acesso múltiplo com desvanecimento Rayleigh e AWGN. O canal foi modelado como uma concatenação de um MAC não ruidoso com um canal ruidoso. Para obter estes resultados foi necessário derivar as densidades de probabilidade que descrevem o canal ruidoso. Obtivemos como resultado que a distribuição de entrada comum a todos os usuários que maximiza a capacidade soma é a distribuição uniforme. Mostramos que um limitante superior simples é próximo a valores obtidos numericamente. Um limitante inferior simples para $T \cdot C_{S U D}$ também foi dado.

\section{AgRAdeCIMENTOS}

Gostaríamos de agradecer aos professores Max Costa e Paulo Valente pelas frutíferas discussões.

\section{APÊNDICE I}

ESCOLHA DA DISTRIBUIÇÃO DE PROBABILIDADE DOS SÍMBOLOS DE ENTRADA QUE ATINGE A CAPACIDADE SOMA

Seja $I=\mathcal{I}(\mathbf{M}, \mathbf{R})$ a informação mútua entre os símbolos de entrada $\mathbf{M}$ e a saída $\mathbf{R}$. Ela pode ser escrita como:

$$
I=\sum_{\mathbf{c} \in \Gamma(c)} P(\mathbf{c}) \int_{\mathbf{R}} p(\mathbf{R} \mid \mathbf{c}) \log _{2}\left[\frac{p(\mathbf{R} \mid \mathbf{c})}{\sum_{\mathbf{c}^{\prime} \in \Gamma(\mathbf{c})} P\left(\mathbf{c}^{\prime}\right) p\left(\mathbf{R} \mid \mathbf{c}^{\prime}\right)}\right] d \mathbf{R}
$$

Suponha que todos os usuários utilizam a mesma distribuição de probabilidade dos símbolos. Então, $P(\mathbf{c})$ é dada por:

$$
P(\mathbf{c})=\frac{T !}{c_{1} ! c_{2} ! \ldots c_{N} !} \mu_{1}^{c_{1}} \mu_{2}^{c_{2}} \ldots \cdot \mu_{N}^{c_{N}}
$$

É claro que $I$ é uma função de $\mu_{1}, \mu_{2}, \ldots, \mu_{N}$. Seja $f_{i}\left(\mu_{1}, \mu_{2}, \ldots, \mu_{N}\right)$ as derivadas parciais $\frac{\partial I}{\partial \mu_{i}}$. Podemos ver da equação (7) que uma permutação nos índices das freqüências não altera as densidades de probabilidade das transições do canal, $p\left(R_{n} \mid c_{n}\right)$. Logo, $f_{i}\left(\mu_{1}, \mu_{2}, \ldots, \mu_{N}\right)$ tem a mesma forma para todo $i$, exceto por uma troca de índices.

A capacidade soma pode ser formulada como o seguinte problema de maximização:

$$
C_{S}=\max _{\mu_{1}, \mu_{2}, \ldots, \mu_{N}} I\left(\mu_{1}, \mu_{2}, \ldots, \mu_{N}\right)-\lambda\left(\mu_{1}+\mu_{2}+\cdots+\mu_{N}-1\right)
$$

de onde obtemos:

$$
\begin{gathered}
\frac{\partial I}{\partial \mu_{1}}=f_{1}\left(\mu_{1}, \mu_{2}, \ldots, \mu_{N}\right)-\lambda \\
\frac{\partial I}{\partial \mu_{2}}=f_{2}\left(\mu_{1}, \mu_{2}, \ldots, \mu_{N}\right)-\lambda \\
\vdots \\
\frac{\partial I}{\partial \mu_{N}}=f_{N}\left(\mu_{1}, \mu_{2}, \ldots, \mu_{N}\right)-\lambda .
\end{gathered}
$$

Concluindo, a solução deve satisfazer:

$$
\lambda=f_{1}(\cdots)=f_{2}(\cdots)=\cdots=f_{N}(\cdots)
$$

Dado os mesmos argumentos, os valores de $f_{1}, f_{2}, \ldots, f_{N}$ são os mesmos. Assim sendo, a solução é $\mu_{1}=\mu_{2}=\ldots=$ $\mu_{N}=1 / N$.

Embora a expressão de $P(\mathbf{c})$ seja mais complexa, este resultado permanece válido mesmo que não consideremos que todos os usuários utilizem a mesma distribuição de entrada.

\section{APÊNDICE II}

\section{ESCOLHA DA DISTRIBUIÇÃO DE PROBABILIDADE DOS SÍMBOLOS DE ENTRADA QUE ATINGE A $C_{S U D}$}

Seja $I=\mathcal{I}\left(m^{j}, \mathbf{R}\right)$ a informação mútua entre os símbolos de entrada $m^{j}$ e a saída $\mathbf{R}$. Ela pode ser escrita como:

$$
I=\sum_{m^{j}=0}^{N-1} P\left(m^{j}\right) \int_{\mathbf{R}} p\left(\mathbf{R} \mid m^{j}\right) \log _{2}\left[\frac{p\left(\mathbf{R} \mid m^{j}\right)}{\sum_{m^{j^{\prime}}=0}^{N-1} P\left(m^{j^{\prime}}\right) p\left(\mathbf{R} \mid m^{j^{\prime}}\right)}\right]
$$

onde $P\left(m^{j}=n\right)=\mu_{n}$.

Novamente, as derivadas parciais em relação à $P\left(m^{j}\right)$ são similares exceto por uma troca de índices, e o mesmo procedimento e resultado do Apêndice I são válidos.

\section{REFERÊNCIAS}

[1] Shih-Chun Chang and J. K. Wolf., On the T-User M-Frequency Noiseless Multiple-Access Channel with and Without Intensity Information, IEEE Transactions on Information Theory, v. 27, n. 1, pp. 41-48, Janeiro de 1981.

[2] O. Yue, Maximum Likelihood Combining for Noncoherent and Differentially Coherent Frequency-Hopping Multiple-Acces Systems, IEEE Transactions on Information Theory, v. 28, n. 4, pp. 631-639, Julho de 1982.

[3] O. Yue, Performance of Frequency-Hoppinf Multiple-Access Multilevel FSK with Hard-Limited and Linear Combining, IEEE Transactions on Communications, v. 29, n. 11, pp. 1687-1694, Novembro de 1981. 